\title{
A single nucleotide substitution in the coding region of Ogura male sterile gene, orf138, determines effectiveness of a fertility restorer gene, $R f o$, in radish
}

\author{
Hiroshi Yamagishi ${ }^{1}$ (D) Megumi Jikuya ${ }^{1} \cdot$ Kanako Okushiro $^{1} \cdot$ Ayako Hashimoto $^{2} \cdot$ Asumi Fukunaga $^{1} \cdot$ \\ Mizuki Takenaka ${ }^{3} \cdot$ Toru Terachi $^{1}$
}

Received: 13 January 2021 / Accepted: 15 March 2021 / Published online: 26 March 2021

(C) The Author(s) 2021

\begin{abstract}
Cytoplasmic male sterility (CMS) observed in many plants leads defect in the production of functional pollen, while the expression of CMS is suppressed by a fertility restorer gene in the nuclear genome. Ogura CMS of radish is induced by a mitochondrial orf138, and a fertility restorer gene, $R f o$, encodes a P-type PPR protein, ORF687, acting at the translational level. But, the exact function of ORF687 is still unclear. We found a Japanese variety showing male sterility even in the presence of $R f o$. We examined the pollen fertility, Rfo expression, and orf $138 \mathrm{mRNA}$ in progenies of this variety. The progeny with Type $\mathrm{H}$ orf 138 and Rfo showed male sterility when their orf138 mRNA was unprocessed within the coding region. By contrast, all progeny with Type A orf 138 were fertile though orf138 mRNA remained unprocessed in the coding region, demonstrating that ORF687 functions on Type A but not on Type H. In silico analysis suggested a specific binding site of ORF687 in the coding region, not the 5' untranslated region estimated previously, of Type A. A single nucleotide substitution in the putative binding site diminishes affinity of ORF687 in Type $\mathrm{H}$ and is most likely the cause of the ineffectiveness of ORF687. Furthermore, fertility restoration by RNA processing at a novel site in some progeny plants indicated a new and the third fertility restorer gene, $R f s$, for orf138. This study clarified that direct ORF687 binding to the coding region of orfl 38 is essential for fertility restoration by $R f o$.
\end{abstract}

Keywords Fertility restorer gene $\cdot$ Ogura cytoplasmic male sterility $\cdot$ Orf138 $\cdot$ ORF687 $\cdot$ PPR-code $\cdot$ Radish

\section{Introduction}

Cytoplasmic male sterility (CMS) that presents a defect in the production of functional pollen is spread in more than 140 plant species (Laser and Lersten 1972). The CMS genes are produced by recombination of the mitochondrial genome and consist of novel open reading frames (orfs) (Hanson and Bentolila 2004). The orfs are translated into

Communicated by Stefan Hohmann.

Hiroshi Yamagishi

hiyamagi@cc.kyoto-su.ac.jp

1 Faculty of Life Sciences, Kyoto Sangyo University, Kamigamo, Kita, Kyoto 603-8555, Japan

2 Research Center of Botany, Kyoto Sangyo University, Kamigamo, Kita , Kyoto 603-8555, Japan

3 Graduate School of Science, Kyoto University, Kitashirakawa-Oiwakecho, Sakyo-ku, Kyoto 606-8502, Japan novel mitochondrial proteins resulting failure to produce functional pollen (Chen and Liu 2014). CMS is suppressed by nuclear genes called fertility restorer genes (Rf genes). Recent studies revealed that a majority of the proteins encoded by Rf genes belongs to the pentatricopeptide repeat (PPR) family. The functions of PPR proteins involve RNA cleavage, RNA destabilization, or translational inhibition (Dahan and Mireau 2013). Thus, the CMS-Rf systems are very important to understand the interactions between the nuclear and mitochondrial genomes in plants. Among the systems, Ogura CMS of radish is one of the most deeply studied systems because the CMS gene, orf138, and an Rf gene, $R f o$, were identified (Gaborieau et al. 2016). However, the exact functional relationship between the two genes is still unclear.

Besides the importance in the biological studies, the CMS trait has been the most effective genetic tool for plant breeding $\left(\mathrm{F}_{1}\right.$ breeding $)$ with the goal of hybrid vigor. CMS provides an efficient and stable $\mathrm{F}_{1}$ seed production system 
for plant breeding because it naturally avoids self-pollination of seed parent plants. Brassicaceae crops show marked hybrid vigor and $F_{1}$ breeding using Ogura CMS, which was originally found in a Japanese radish (Ogura 1968), has been conducted worldwide not only in radishes but also in various Brassica crops including Brassica oleracea, Brassica napus, and Brassica juncea. As aforementioned, the causal gene of the Ogura CMS is a mitochondrial gene, orf138 (Bonhomme et al. 1991), which is located at the $5^{\prime}$ region of atp 8 and expressed as a bicistronic transcript together with atp 8 (Bonhomme et al. 1992; Krishnasamy and Makaroff 1993).

We observed that orf138 is distributed in more than $40 \%$ of Japanese wild radishes (Yamagishi and Terachi 1996). Sequence analyses of orf138 in large numbers of wild and cultivated radishes revealed six nucleotide substitutions and one insertion/deletion in the coding region of the gene (Yamagishi and Terachi 2001). Using the combination patterns of the seven nucleotide-variations, orfl 38 were classified into nine haplotypes, Type A-I. We inferred that Type $\mathrm{B}$ is the ancestral form of the orf 138 from the comparison of the nucleotide variations among the nine types (Yamagishi and Terachi 2001). The Type B orf 138 was found in a wild species, Raphanus raphanistrum, as a major type, and it is linked with male sterility (Yamagishi and Terachi 1997, 2001). On the other hand, the same Type B orfl38 has also been found in European natural populations of $R$. raphanistrum, but surprisingly, it did not induce male sterility (Giancola et al. 2007), suggesting that at least one fertility restorer gene ( $\mathrm{Rf}$ gene) for Type B orfl38 is widely spread in European natural population.

The phenotypic expression of male sterility is controlled by combinations of mitochondrial CMS genes and $\mathrm{Rf}$ gene(s), encoded in the nuclear genome. An Rf gene for Ogura CMS was identified by three groups and named Rfo or orf687 (Brown et al. 2003; Desloire et al. 2003; Koizuka et al. 2003). This gene (hereafter, Rfo) alters expression of $\operatorname{orf} 138$ at a post-transcriptional level (Desloire et al. 2003 ) and either directly or indirectly lowers the amount of ORF138 protein (Koizuka et al. 2003). Rfo encodes a 687 amino acid pentatricopeptide repeat (PPR) protein referred to as ORF687. ORF687 is thought to interact with the orf138 mRNA, or to act on the post-translational stability of the ORF138 protein, since the amount and sequence of $\operatorname{orfl} 138$ mRNA was not altered by the presence of ORF687 (Bellaoui et al. 1999; Uyttewaal et al. 2008). Although Uyttewaal et al. (2008) proposed that ORF687 could associate with the 5' untranslated region (5' UTR) of orf $138 \mathrm{mRNA}$, the function of ORF687 to prevent the translation is still unclear. While, Koizuka et al. (2003) observed four amino acid substitutions in the second and third repeats of the PPR domain between an $r f o$ allele in male sterile lines and an Rfo allele in fertilityrestored lines. They inferred that the two PPR repeats in ORF687 are essential for fertility restoration (Koizuka et al.
2003). Furthermore, Imai et al. (2002) found that the 118th amino acid in the PPR protein among the four substitutions plays a crucial role for fertility restoration.

Based on these findings, we surveyed the distribution of the $R f o$ allele in Japanese wild radishes. However, the frequency of the $R f o$ allele was unexpectedly low, with about $15 \%$ plants possessing this allele though more than $90 \%$ plants had one or more Rf gene(s) (Yasumoto et al. 2008). Therefore, we searched for additional Rf genes and found a novel gene that works in the processing of orf $138 \mathrm{mRNA}$ in the Japanese wild radish population and named it $R f t$ (Yasumoto et al. 2009), although the gene has not yet been cloned. By the product of $R f t$, orfl 38 transcript lost part of the $5^{\prime}$ coding region before the 102nd-104th nucleotides from the start codon (Yasumoto et al. 2009).

Our results indicated that at least nine types of orf 138 are distributed in wild and cultivated radishes, and that two or more Rf genes are present in radishes. However, the correlations between the various types of orf 138 and the effect of multiple Rf genes have not been well studied. In this report, we surveyed the types of orf 138 and the distribution of $R f o$ and $R f t$ in commercially available modern cultivars in Japan. We also determined the DNA sequences of orf138 as well as $R f o$ genes in respective cultivars. Furthermore, expressions of these genes and male sterility phenotypes in progeny produced by cross-hybridizations among the cultivars were compared. The results revealed interesting relationships between the orf138 types and the function of ORF687. The validity of ORF687 toward orfl38 is disrupted by a single nucleotide substitution in the coding region of orf 138 where ORF687 binds directly to the orf 138 mRNA. Our data also suggest that another Rf gene other than $R f o$ and $R f t$ is present in radishes. The new $\mathrm{Rf}$ gene named $R f s$ processes orf 138 mRNA at a different position from that of $R f t$.

\section{Materials and methods}

\section{Plant materials}

In our preliminary study, we found a radish variety, 'BK', which shows male sterility and possesses RfoRfo genotype. We employed 'BK' and 'MR', which showed the rforfo genotype and male sterility, for cross hybridization. We also used two cultivars 'MS-Gensuke' ('MS-G' in this article) and 'Soubaika Kansaitou' ('SK' in this article) that possesses Type A and Type H orf138, respectively (Yamagishi and Terachi 2001). 'MS-G' is a male sterile variety that we use as a control of the Ogura CMS. 'SK' showed normal pollen fertility, and the fact suggested that this variety encodes at least one Rf gene. We crossed 'BK' and 'SK' first. Using one hybrid plant of the cross, ('BK' $\times$ 'SK'), as a common pollen parent, cross hybridization was carried out 
with 'MR' or 'MS-G' as a female parent and the progenies were used for further analyses. The radish plants were grown in a glass house.

\section{DNA and RNA isolation}

Total DNA was isolated from young leaves using a DNeasy Plant Mini Kit (QIAGEN, California, USA). Total RNA was isolated from flower buds (about $0.1 \mathrm{~g}$ ) using an RNeasy Plant Mini Kit (QIAGEN).

\section{Examination of Rfo genotypes and the presence of Rft locus}

To investigate whether the plants had a DNA sequence encoding functional ORF687 (Rfo) or had the rforfo genotype, which lacks the Rf function, we performed PCR-RFLP analysis with a primer pair and the restriction enzyme $S s p \mathrm{I}$ as described (Yasumoto et al. 2008). The presence of $R f t$ was identified using the STS marker, following the reported procedure (Yasumoto et al. 2009). All primers used in this experiment, including those used to examine $R f o$ and $R f t$ are shown in Table S1.

\section{Sequencing of orf 138 and $R f o$}

PCR products containing an entire coding and the 5'/3'-flanking regions of orfl38 were purified using a QIAquick PCR purification Kit (QIAGEN) and directly sequenced using a CEQ 8000DNA sequencer (Beckman Coulter, California, USA). The DNA sequences of $R f o$ were determined similarly, using a PCR product amplified with a primer pair described in Yasumoto et al. (2009). The amino acid sequence of ORF687 was deduced based on the DNA sequence.

\section{Northern blot of orf138 and qRT-PCR of Rfo in fertile and sterile progeny plants}

Northern blot analysis for orf 138 mRNA was conducted using total RNA isolated from flower buds according to the reported method (Yasumoto et al. 2009). Total RNA was hybridized with the probe located over the entire coding region of orf138 (Probe I in Yasumoto et al. 2009). To examine the expression of $R f o$, quantitative RT-PCR (qRTPCR) was carried out. To do this, total RNA was extracted from the flower buds of three fertile and three sterile plants among the progeny of 'MR' $\times($ 'BK' $\times$ 'SK'). After cDNA was synthesized, quantitative PCR was carried out using KOD SYBR ${ }^{\circledR}$ qPCR Mix (TOYOBO, Osaka, Japan) with 7500 Real Time PCR system (Applied Biosystems, Massachusetts, USA). Actinl was used as an internal control for RNA expression.
Detection of the processing site in the orf138 mRNA

Circular RT-PCR was conducted with the methods described in Perrin et al. (2004) and Giancola et al. (2007). Briefly, total RNA was incubated with T4 RNA ligase (New England Biolabs, MA, USA), and cDNA was synthesized. The region containing the junction of $5^{\prime}$ and $3^{\prime}$ extremities was amplified by RT-PCR. The circular RT-PCR products were cloned using the PGem ${ }^{\circledR}$ - TEasy Vector Systems (Promega, Madison, USA), and the sequences were determined. The primers used in the procedure are shown in Table S1.

\section{Prediction of target site of ORF687}

To predict the potential binding sites for the ORF687 variants from 'BK', 'SK', and 'MR', we used the FIMO program in the MEME suite (http://meme-suite.org/tools/fimo). We generated a putative nucleotide binding motif for ORF687 using the identities of the amino acids at the 5th and last position of each PPR motif. These nucleotide preference scores (Figure $\mathrm{S} 1$ ) were used to predict the best potential binding site search for ORF687 in Type A or Type $\mathrm{H}$ of orf138 mRNA sequences using the FIMO program. The predicted binding sites were ranked by $P$ values calculated by FIMO.

\section{Results}

\section{A male sterile radish variety ' $\mathrm{BK}$ ', as well as a male fertile 'SK', has Type H orf138}

More than 10 male sterile Japanese radish varieties were observed by our preliminary survey and all of them possessed orf138. Although most of them had rforfo genotype at the $R f o$ locus, one of the male sterile cultivars named 'BK' possessed the RfoRfo genotype. We focused on 'BK' because this could provide a clue for understanding the molecular mechanism of $R f o$-mediated fertility restoration. First, we ascertained that 'BK' and two other male sterile varieties, 'MS-G', 'MR', and a male fertile variety, 'SK', possess orf 138 by PCR (Fig. 1a). Next, the DNA sequences of all the coding and $5^{\prime} / 3^{\prime}$ flanking regions of orf 138 were determined for 'MR', 'BK', and 'SK'. The three cultivars had Type $\mathrm{H}$ orfl 38 (Table 1). In Type $\mathrm{H}$, the 61st nucleotide from the AUG start codon of orf138 is $\mathrm{C}$ whereas that in Type A ('MS-G') and Type B is A instead. The Type $\mathrm{H}$ has $\mathrm{G}$ at the 99th nucleotide of orf138 as well as Type B, while Type A ('MS-G') has A (Yamagishi and Terachi 2001). Table 2 shows the nucleotide substitutions among the three haplotypes. Besides these two sites, the sequences of the orf138 in 'MR' and 'BK' were identical to Type A ('MS-G'), in correlation to our previous results (Yamagishi 
Fig. 1 Presence of orf138 and $R f t$, and genotyping of $R f o$ locus. a Detection of orf138 DNA. b PCR-RFLP of $R f o$ locus to determine the genotype. c Detection of $R f t$ by the STS marker. 1: 'Uchiki-Gensuke' (a maintainer of Ogura CMS). 2: 'MS-G'. 3: 'MR'. 4: 'BK'. 5:

'SK'. C1: A Japanese wild radish with RfoRfo genotype. C2: A Japanese wild radish having $R f t$ a

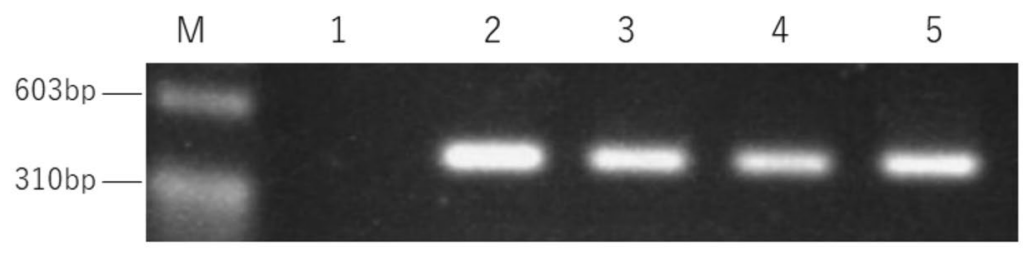

b

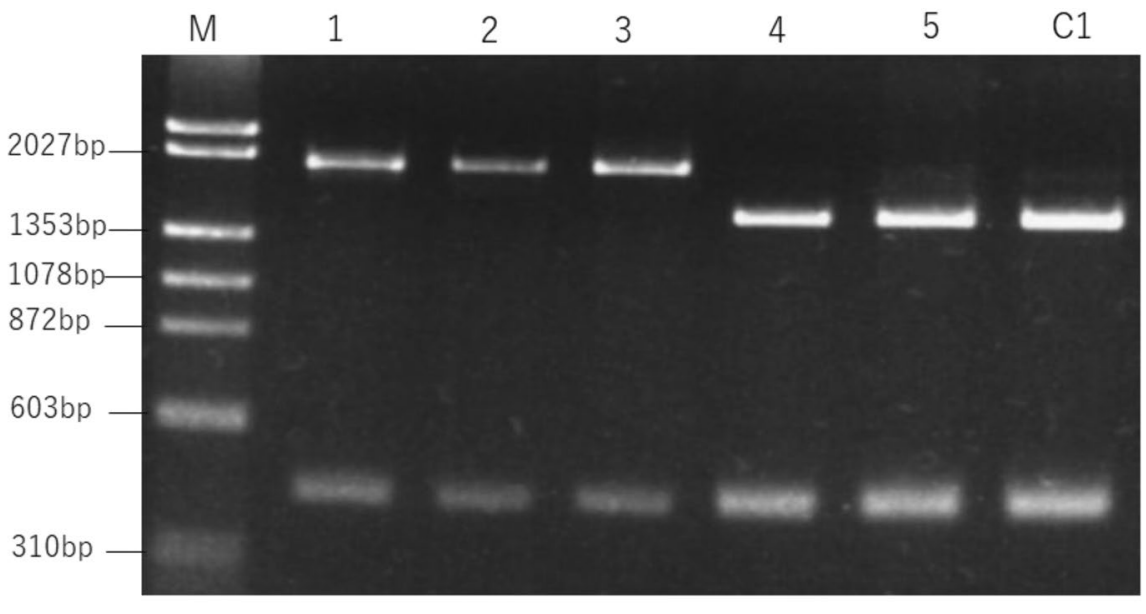

c

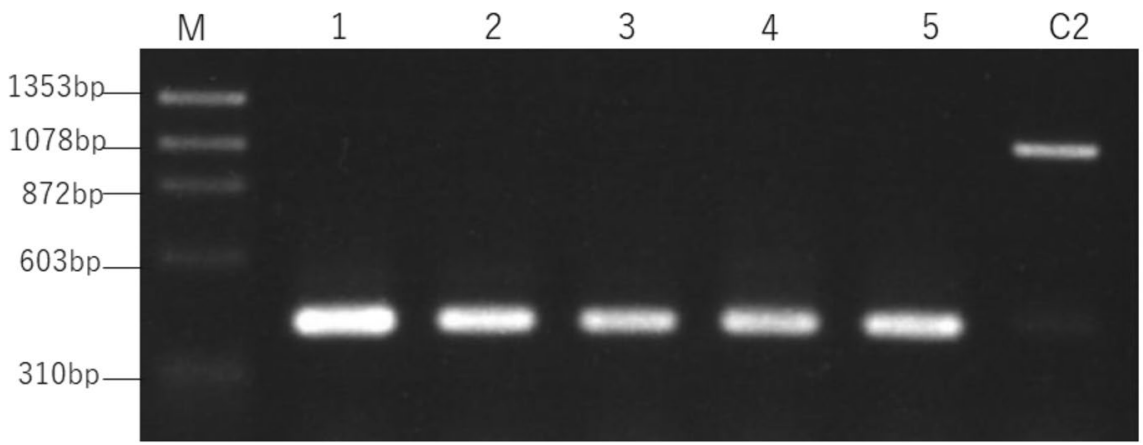

Table 1 Type of orf138, genotypes and presence of Rf genes, and pollen fertility of radish cultivars

\begin{tabular}{|c|c|c|c|c|}
\hline \multirow[t]{2}{*}{ Cultivars } & \multirow{2}{*}{$\begin{array}{l}\text { Type of } \\
\text { orf138 }\end{array}$} & \multicolumn{2}{|l|}{ Rf genes } & \multirow[t]{2}{*}{ Pollen fertility } \\
\hline & & $R f o$ genotype & $R f t$ & \\
\hline MR & $\mathrm{H}$ & rfo rfo & $-^{\mathrm{a}}$ & Sterile \\
\hline BK & $\mathrm{H}$ & Rfo Rfo & - & Sterile \\
\hline SK & $\mathrm{H}$ & Rfo Rfo & - & Fertile \\
\hline MS-G & A & rfo rfo & - & Sterile \\
\hline
\end{tabular}

a $-R f t$ is absent

Table 2 Nucleotide substitutions in the coding region of orf138 among the three haplotypes

\begin{tabular}{lll}
\hline Haplotype & $\begin{array}{l}\text { Nucleotide } \\
61\end{array}$ & $\begin{array}{l}\text { Sites } \\
99\end{array}$ \\
\hline Type A & A & A \\
Type B & A & G \\
Type H & C & G \\
\hline
\end{tabular}

and Terachi 2001). Furthermore, Type A and Type $\mathrm{H}$ did not show any differences in the intergenic region between trnfM and $\operatorname{orf138}$ (data not shown).

\section{Distribution of $R f o$ and $R f t$ genes}

A nucleotide variation inducing the substitution of the 118th amino acid of ORF687 was used as an RFLP marker (Yasumoto et al. 2008) and the genotypes of the $R$ fo locus were estimated. 'MR' and 'MS-G' showing male sterile phenotype and lacking $\mathrm{Rf}$ genes had the genotype of rforfo at the Rfo locus, while 'SK' with normal pollen fertility showed $R$ foRfo genotype as expected (Table 1, Fig. 1b). However, 'BK' showed the RfoRfo genotype despite that it exhibited the male sterile phenotype and had Type H orf138, as well as 'MR' (Table 1, Fig. 1b). In addition to the genotype at the $R f o$ locus, we examined the presence of the $R f t$ locus with a STS marker. All the varieties analyzed here showed the DNA amplification pattern 
Table 3 Amino acid substitutions in ORF687 among the four analyzed radish cultivars

\begin{tabular}{lllll}
\hline Cultivars & \multicolumn{3}{l}{$\begin{array}{l}\text { Amino acid positions } \\
\text { in ORF687 }\end{array}$} \\
\cline { 2 - 5 } & 118 & 153 & 170 & 171 \\
\hline MR & T & N & N & L \\
BK & N & T & D & F \\
SK & N & T & D & F \\
MS-G & T & N & N & L \\
\hline
\end{tabular}

Table 4 Pollen fertility and transcript pattern of orf138 in the progeny of 'MR' $\times($ 'BK' $\times$ 'SK')

\begin{tabular}{llccc}
\hline Pollen fertility & \multicolumn{4}{l}{ Transcript pattern } \\
\cline { 2 - 5 } & $1.4-\mathrm{kb}$ & $1.4-\mathrm{kb} / 1.1-\mathrm{kb}$ & $1.1-\mathrm{kb}$ & Total \\
\hline Fertile & 0 & 0 & 11 & 11 \\
Sterile & 8 & 14 & 0 & 22 \\
Total & 8 & 14 & 11 & 33 \\
\hline
\end{tabular}

\section{Another $\mathrm{Rf}$ gene other than $\boldsymbol{R f o}$ and $\boldsymbol{R f t}$ restored male fertility}

'BK' and 'SK' had identical characteristics in all aspects examined here except for pollen fertility; namely, the two varieties had Type H orf138, RfoRfo genotype, and lacked $R f t$ (Table 1). To investigate the difference between the two varieties, we crossed them and hybrid plants were obtained. One of the hybrid plants, ('BK' $\times$ 'SK'), was used to pollinate both 'MR' and 'MS-G'. Among the characteristics we investigated, the only difference between 'MR' and 'MS-G' was the type of orf138 (Table 1), 'MR' has Type H while 'MS-G' has Type A. The progeny of the cross ' $\mathrm{MR}$ ' $\times$ ('BK' $\times$ 'SK') was segregated into 11 fertile and 22 sterile plants (Table 4). The orf138 mRNA of the 11 fertility-restored progenies was processed in the coding region of orf138, showing about $1.1 \mathrm{kbp}$ in size (Table 4, Fig. 2), even though they lacked $R f t$. In the sterile plants, the orf138 mRNA was either partially processed (14 plants) or had the full-length

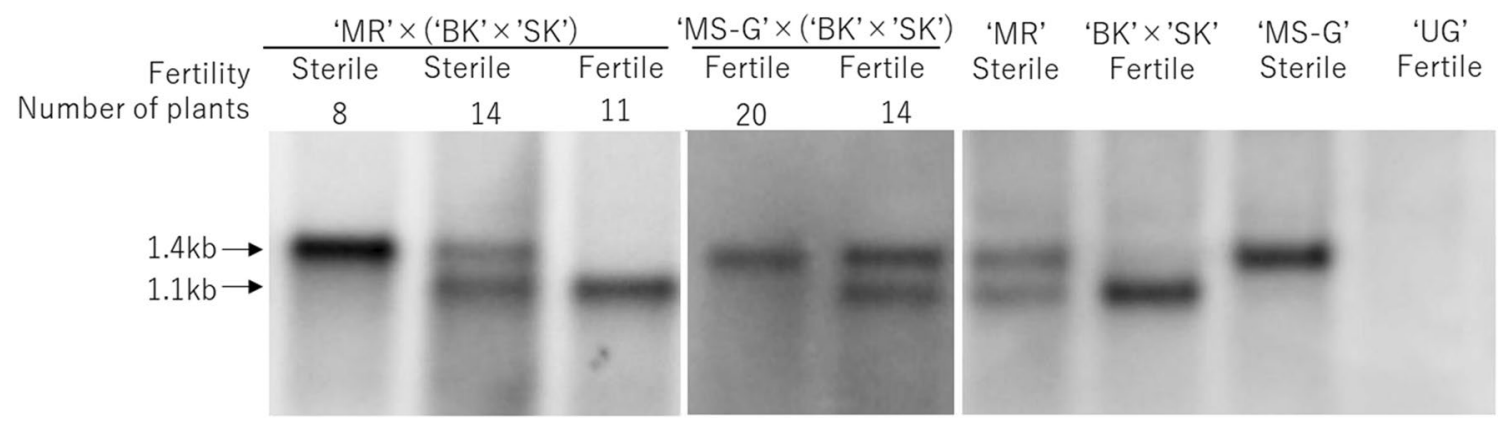

Fig. 2 Transcript patterns of orf138 and pollen fertility in the progenies of 'MR' $\times($ 'BK' $\times$ 'SK') and 'MS-G' $\times($ 'BK' $\times$ 'SK'). 'UG'; the maintainer of 'MS-G' ('Uchiki-Gensuke') used as a negative control

of one lacking $R f t$ (Table 1, Fig. 1c), indicating that the fertility restoration of ' $\mathrm{SK}$ ' was not by the product of $R f t$.

\section{Amino acid sequence of ORF687 was identical between 'BK' and 'SK'}

Unexpected male sterility in ' $\mathrm{BK}$ ' could be due to additional amino acid substitutions in ORF687 other than the 118 th amino acid. Thus, we analyzed the DNA sequence of $R f o$ genes. The predicted amino acid sequences of ORF687 were completely identical between 'BK' and 'SK' despite the difference in their phenotypes (Table 3). The two cultivars ('MR' and 'MS-G') that showed rforfo genotype for the RFLP site mentioned above commonly had four different sites from ' $\mathrm{BK}$ ' and ' $\mathrm{SK}$ ' including the 118th amino acid (Table 3).
Table 5 Pollen fertility and transcript pattern of orf 138 in the progeny of 'MS-G' $\times$ ('BK' $\times$ 'SK')

\begin{tabular}{lcclc}
\hline Pollen fertility & \multicolumn{4}{l}{ Transcript pattern } \\
\cline { 2 - 5 } & $1.4-\mathrm{kb}$ & $1.4-\mathrm{kb} / 1.1-\mathrm{kb}$ & $1.1-\mathrm{kb}$ & Total \\
\hline Fertile & 20 & 14 & 0 & 34 \\
Sterile & 0 & 0 & 0 & 0 \\
Total & 20 & 14 & 0 & 34 \\
\hline
\end{tabular}

orf138 mRNA (8 plants) (Table 4, Fig. 2). This result indicated that the Type $\mathrm{H}$ orf138 of 'MR' induced male sterility when the mRNA was not completely processed in the coding region, even though the genotype of the Rfo locus was Rforfo in all progeny plants. In contrast, all of the progeny plants from the cross ' $\mathrm{MS}-\mathrm{G}$ ' $\times($ ' $\mathrm{BK}$ ' $\times$ ' $\mathrm{SK}$ ') had normal pollen fertility (Table 5). Moreover, the orf138 mRNA in these 
34 progeny plants was either partially processed (14 plants) or not processed in the coding region (20 plants) (Table 5, Fig. 2). Pollen fertility was restored in all the progeny plants despite the fact that orf 138 mRNA was not perfectly processed in the coding region in the progeny of 'MS-G'. The results indicated that the $R f o$ functions on Type A orf138. The expression of orf 138 in the presence of $R f o$ depended on whether the orf138 was Type A or Type H. The expression of Type A orf138 ('MS-G') was suppressed even though the mRNA was kept in full-length. On the other hand, Type $\mathrm{H}$ orf138 ('MR') caused male sterility when the mRNA was not processed in the coding region.

\section{Rfo was expressed in both the fertile and sterile progeny plants}

To verify that the ineffectiveness of $R f o$ in the male sterile plants with Type $\mathrm{H}$ was not due to repression of the $R f o$ gene, the transcription of $R f o$ gene was investigated in fertile and sterile plants in the progeny of the cross 'MR' $\times($ 'BK' $\times$ 'SK'). The ratio of transcribed mRNA of $R f o$ gene to that of actinl analyzed by qRT-PCR demonstrated high amounts of $R f o$ transcription both in the fertile and the sterile progenies (Table 6). The transcription level in the sterile plants was higher than that in the fertile plants (126.7\%) (Table 6), but the difference was not statistically significant $(t=0.385)$, indicating that $R f o$ was expressed in the sterile plants at levels similar to those observed in fertile plants.

\section{Processing site of orf138 mRNA}

The Northern blot analysis demonstrated that all the fertile plants in the progeny of the cross ' $\mathrm{MR}$ ' $\times\left({ }^{\prime} \mathrm{BK}^{\prime} \times\right.$ ' $\mathrm{SK}$ ') showed complete processing of orf $138 \mathrm{mRNA}$ in the coding region (Table 4). Since the observed RNA processing was independent from the presence of the $R f t$ gene, we conducted circular RT-PCR to determine the exact processing site in orf1 38 mRNA. From 8 plants resulting from the cross 'MR' $\times($ 'BK' $\times$ 'SK'), 4 of which were fertile and 4 were sterile,

Table 6 Transcription of $R f o$ in the fertile and sterile plants of the progeny of 'MR' $\times($ 'BK $\times$ 'SK')

\begin{tabular}{ll}
\hline Pollen fertility & $\begin{array}{l}\text { Transcription } \\
\text { relative to } \\
\text { actinl }\end{array}$ \\
\hline Fertile (A) & $6.15 \pm 1.00^{\mathrm{b}}$ \\
Sterile (B) & $7.79 \pm 4.15^{\mathrm{b}}$ \\
Ratio (B/A) & $126.7 \%$ \\
\hline
\end{tabular}

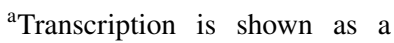
relative ratio to actinl used as an internal control for RNA expression

${ }^{\mathrm{b}}$ The data are shown as mean \pm SD of three plants
24 clones were obtained. The majority of the clones (22 of 24 clones) had a $3^{\prime}$ end corresponding to the $3^{\prime}$ end of the orf138-atp 8 co-transcript irrespective of pollen fertility (Fig. 3). The precise 3' ends of the clones was at the 120th nucleotide downstream of atp 8 ; that is 8 nucleotides downstream of the 3' end shown by Bonhomme et al. (1992), and 9 nucleotides upstream of that indicated by Bellaoui et al. (1999). The six clones obtained from the three sterile plants had $5^{\prime}$ ends at +1 (four clones) or +3 (two clones) downstream of the trnfM coding sequence and a full-length orf 138 mRNA (Fig. 3). The 5 ' termini were identical to those previously reported (Bellaoui et al. 1999; Yasumoto et al. 2009). The other 8 clones derived from the sterile plants and nine clones from the fertile plants had $5^{\prime}$ ends ranging from the $143 \mathrm{rd}$ to the $183 \mathrm{rd}$ nucleotide of the coding sequence of orf138; the $5^{\prime}$ ends most frequently observed corresponded to the 156th ( 2 clones), the 157 th ( 5 clones), and the 159th (3 clones) (Fig. 3). Although there was another clone with a $5^{\prime}$ end 17 bp upstream of the site processed by the $R f t$ product, clones with the same $5^{\prime}$ end produced by the processing by $R f t$ products (i.e. the 102 nd-the 104 th nucleotides) were not obtained. Thus, the majority of the orf 138 mRNAs derived from Type $\mathrm{H}$ ('MR') had either the fulllength mRNA or a mRNA that was processed at around the 156th-159th nucleotide.

A total of 32 clones were obtained from the eight plants from the cross 'MS-G' $\times($ ' $\mathrm{BK}$ ' $\times$ 'SK') that were all fertile. For 20 of these clones, the 3 ' ends of the orf138-atp 8 co-transcript were at the same site (the 120th nucleotide) as was found for the progenies of the cross 'MR' $\times($ 'BK' $\times$ 'SK') (Fig. 3), and the 3' end of four clones were placed 1-6 nucleotides further upstream of this position. The other 8 clones had the $3^{\prime}$ termini within the coding sequence of atp8. With regard to the $5^{\prime}$ end, none of these were completely processed in the coding region of orfl38 (Table 5). Twelve clones of the 32 had a $5^{\prime}$ end just downstream of trnfM (five clones at +1 and seven clones at +3 ) (Fig. 3). The $5^{\prime}$ end of 14 clones ranged from the 129th to the 183rd position from the start codon of orf138, and major sites were concentrated in the region containing the 156th ( 2 clones), 157th (3 clones), and 159th ( 2 clones) (Fig. 3). The other six clones had a $5^{\prime}$ end at 34-100 bp upstream of the $R f t$ processing site (five clones) or far downstream of the major $5^{\prime}$ end sites (one clone). Again, no clones had a 5' end at the resulting processed site by the product of $R f t$.

\section{The PPR-code suggested that ORF687 derived from $R$ fo allele prefers to bind to Type A orf138 than to Type $H$}

Association of ORF687 to orf 138 mRNA in vivo was previously demonstrated (Uyttewaal et al. 2008), though its exact binding site has been unclear. The fact that the ORF687 


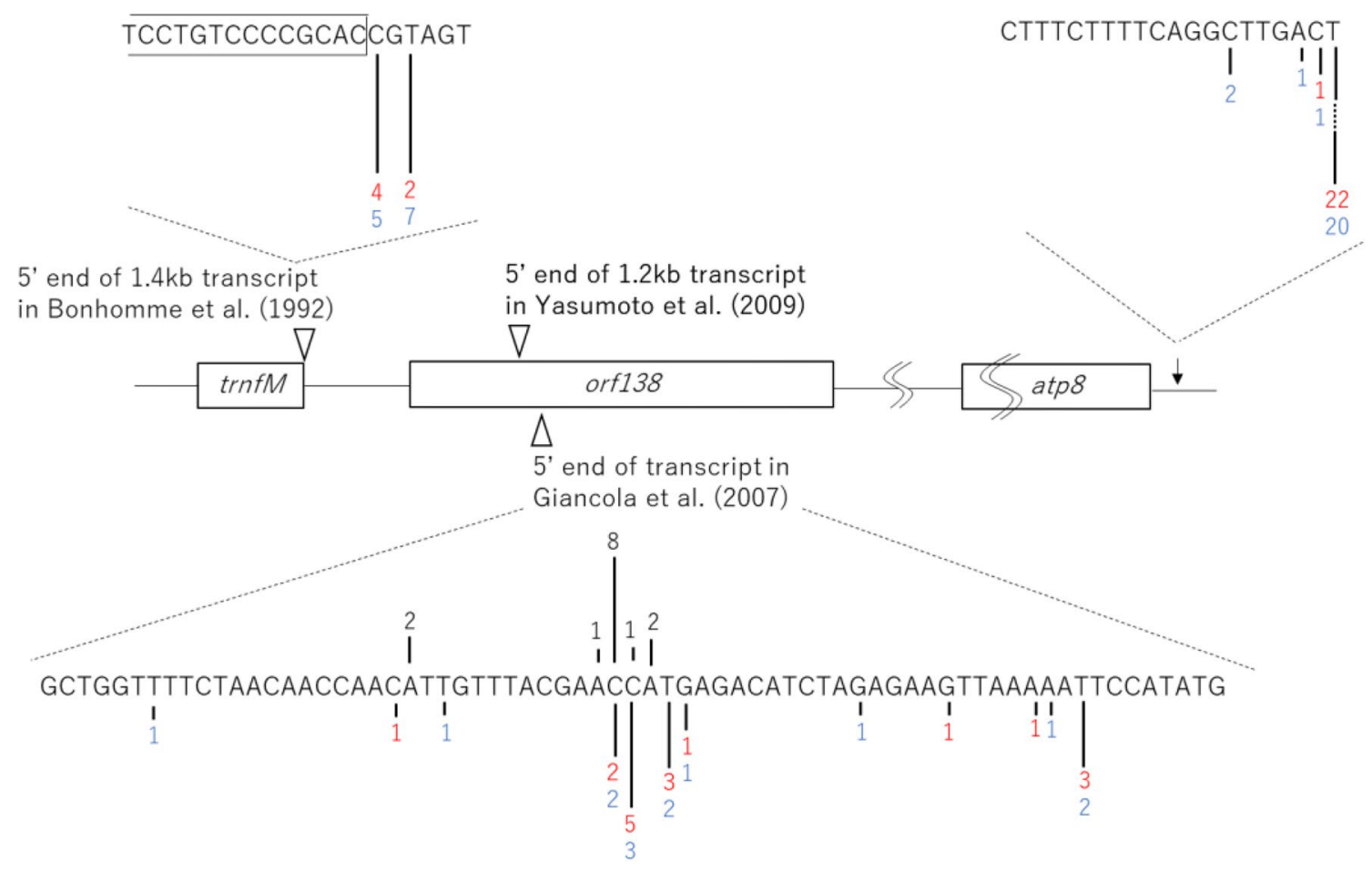

Fig. $35^{\prime}$ and $3^{\prime}$ ends mapping of the orf138 mRNA. Three arrow heads indicate the previously reported $5^{\prime}$ end in different radish varieties. The black arrow indicates the $3^{\prime}$ end of orf138-atp8 co-transcript. Position of $5^{\prime}$ or $3^{\prime}$ ends and their frequency are indicated by

from the Rfo allele functions only to Type A but not to Type $\mathrm{H}$ provided a hint for the exact binding site in the orf138 mRNA. Therefore, we predicted the binding site of ORF687 derived from the Rfo allele in Type A or Type $\mathrm{H}$ orf 138 mRNA using the PPR-code (Yan et al. 2019). ORF687 contains 17 PPR domains (Table 7). Of these, 16 repeats were made up of 35 amino acids and one was 36 amino acids. We searched for the RNA sequence with which ORF687 from $R f o$ allele of 'BK' (hereafter called ORF687-BK) interacts with the highest possibility in the full-length transcript of Type A orf138. The top candidate of the binding sequences was 17 nucleotides (GUAAAGUUAGUGUAAUA) spanning from the 59th to the 75th position in the coding region of Type A orf138 (Table 7, Fig. 4). Interestingly, this sequence contained a nucleotide substitution at the third position between the two orf138 haplotypes, where Type A has A but Type H has C (Tables 2, 7). Target prediction of ORF687BK toward Type $\mathrm{H}$ orf 138 also listed up the same 17 nucleotide sequence as the most probable binding site, though the affinity shown by the difference of $\mathrm{P}$ value decreased from $1.32 \mathrm{E}-06$ with Type A to $6.51 \mathrm{E}-05$ with Type $\mathrm{H}$ (Table 7, Fig. 4). This is because the threonine (T) and asparagine (N) at the two key positions for the PPR code in the third PPR motif of ORF687-BK corresponds exclusively to the nucleotide A, which Type A has but Type $\mathrm{H}$ does not. This bars and numbers, respectively. Red numbers ('MR' $\times($ 'BK' $\times$ 'SK'), Blue numbers: 'MS-G' $\times($ 'BK' $\times$ 'SK'). Black numbers: European wild radish (Giancola et al. 2007). The length of the bars reflects the frequency of each end

is most likely the cause why ORF687 of 'BK' and 'SK' did not function to suppress the translation of Type $\mathrm{H}$ orf 138 mRNA derived from 'MR' when the transcripts were not processed in the coding region (Table 4).

\section{Amino acid substitutions in ORF687 from rfo allele decreased affinity to orf138 mRNA}

The PPR code might also explain the distinct effectiveness between $R f o$ and $r f o$ type ORF687. Thus, we compared the possible target sites of the two types of ORF687 between Type A and Type $\mathrm{H}$ orf 138 . The amino acid variations between the Rfo allele ('BK' and 'SK') and rfo allele ('MR' and 'MS-G') were located at the fifth amino acid of the second PPR (the 118th in Table 3) and the fifth of the third PPR (the 153rd in Table 3). Both of these two sites are crucial for the sequence specificity according to the PPR-code (Table 7), while the amino acid substitutions at the 170th and 171st positions (Table 3) corresponding to the 22nd and 23rd of the third domain are not likely to be involved in the nucleotide recognition. The amino acid substitutions in rfo changed the key amino acid residues for the PPR code from a N-D combination to T-D at the second motif and from $\mathrm{T}-\mathrm{N}$ to $\mathrm{N}-\mathrm{N}$ at the third motif (Table 7). The second repeat with a T-D combination 
Table 7 Alignment of the PPR codes of ORF687 and its putative binding site in the orf 138 mRNA

\begin{tabular}{|c|c|c|c|c|c|c|c|c|}
\hline \multirow[t]{2}{*}{ PPR no. } & \multicolumn{2}{|l|}{ ORF687 of 'BK' } & \multicolumn{2}{|c|}{ orf138 mRNA $^{\mathrm{a}}$} & \multicolumn{2}{|l|}{ ORF687 of 'MR' } & \multicolumn{2}{|c|}{ orf138 mRNA $^{\mathrm{a}}$} \\
\hline & PPR code $\cos ^{\mathrm{b}} 5 \times 35$ & $\begin{array}{l}\text { Nucleotide } \\
\text { preference }\end{array}$ & Type A & Type H & PPR code ${ }^{\mathrm{b}} 5 \times 35$ & $\begin{array}{l}\text { Nucleotide } \\
\text { preference }\end{array}$ & Type A & Type H \\
\hline 1 & $\mathrm{CD}$ & G & G & G & $\mathrm{CD}$ & G & G & G \\
\hline 2 & $\underline{N D}$ & $\mathrm{U}>\mathrm{C}$ & $\mathrm{U}$ & $\mathrm{U}$ & $\underline{T D}$ & $\mathrm{G}>\mathrm{A}$ & $\mathbf{U}$ & $\mathbf{U}$ \\
\hline 3 & $\underline{T N}$ & $\mathrm{~A}>\mathrm{G}$ & $\mathrm{A}$ & $\mathbf{C}$ & $\underline{\mathrm{NN}}$ & $\mathrm{C}>\mathrm{U}$ & $\mathbf{A}$ & $\mathrm{C}$ \\
\hline 4 & $\mathrm{TT}$ & $A>G$ & A & A & TT & $A>G$ & A & A \\
\hline 5 & GN & A & A & A & GN & A & A & A \\
\hline 6 & SD & G & G & G & SD & G & G & G \\
\hline 7 & ND & $\mathrm{U}>\mathrm{C}$ & $\mathrm{U}$ & $\mathrm{U}$ & ND & $\mathrm{U}>\mathrm{C}$ & $\mathrm{U}$ & $\mathrm{U}$ \\
\hline 8 & $\mathrm{NN}$ & $\mathrm{C}>\mathrm{U}$ & $U$ & $U$ & NN & $\mathrm{C}>\mathrm{U}$ & $U$ & $U$ \\
\hline 9 & SN & A & A & A & SN & A & A & A \\
\hline 10 & ND & $\mathrm{U}>\mathrm{C}$ & $\mathbf{G}$ & G & ND & $\mathrm{U}>\mathrm{C}$ & $\mathbf{G}$ & $\mathbf{G}$ \\
\hline 11 & ND & $\mathrm{U}>\mathrm{C}$ & $\mathrm{U}$ & $\mathrm{U}$ & ND & $\mathrm{U}>\mathrm{C}$ & $\mathrm{U}$ & $\mathrm{U}$ \\
\hline 12 & DD & - & $\underline{\mathrm{G}}$ & $\underline{\mathrm{G}}$ & DD & - & $\underline{\mathrm{G}}$ & $\underline{\mathrm{G}}$ \\
\hline 13 & ND & $\mathrm{U}>\mathrm{C}$ & $\mathrm{U}$ & $\mathrm{U}$ & ND & $\mathrm{U}>\mathrm{C}$ & $\mathrm{U}$ & $\mathrm{U}$ \\
\hline 14 & SN & A & A & A & SN & A & A & A \\
\hline 15 & $\mathrm{TN}$ & $\mathrm{A}>\mathrm{G}$ & A & A & $\mathrm{TN}$ & $\mathrm{A}>\mathrm{G}$ & A & A \\
\hline 16 & ID & - & $\underline{\mathrm{U}}$ & $\underline{\mathrm{U}}$ & ID & - & $\underline{\mathrm{U}}$ & $\underline{\mathrm{U}}$ \\
\hline 17 & $\mathrm{RF}$ & $?$ & $\underline{\mathrm{A}}$ & $\underline{\mathrm{A}}$ & $\mathrm{RF}$ & $?$ & $\underline{\mathrm{A}}$ & $\underline{\mathrm{A}}$ \\
\hline & $P$ value & & $1.32 \mathrm{e}-06$ & $6.51 \mathrm{e}-05$ & $P$ value & & $1.04 \mathrm{e}-03$ & $1.18 \mathrm{e}-04$ \\
\hline
\end{tabular}

${ }^{a}$ Normal letters indicate the matches between the PPR-code (nucleotide preference) for each PPR motif and corresponding nucleotide identity. Bold letters indicate the mismatches. Italic letters show the partial matches. The underlined letters indicate the PPR-code is neutral or unclear

b Amino acids with underline indicate the substitution between 'BK' (RfoRfo) and 'MR' (rforfo)

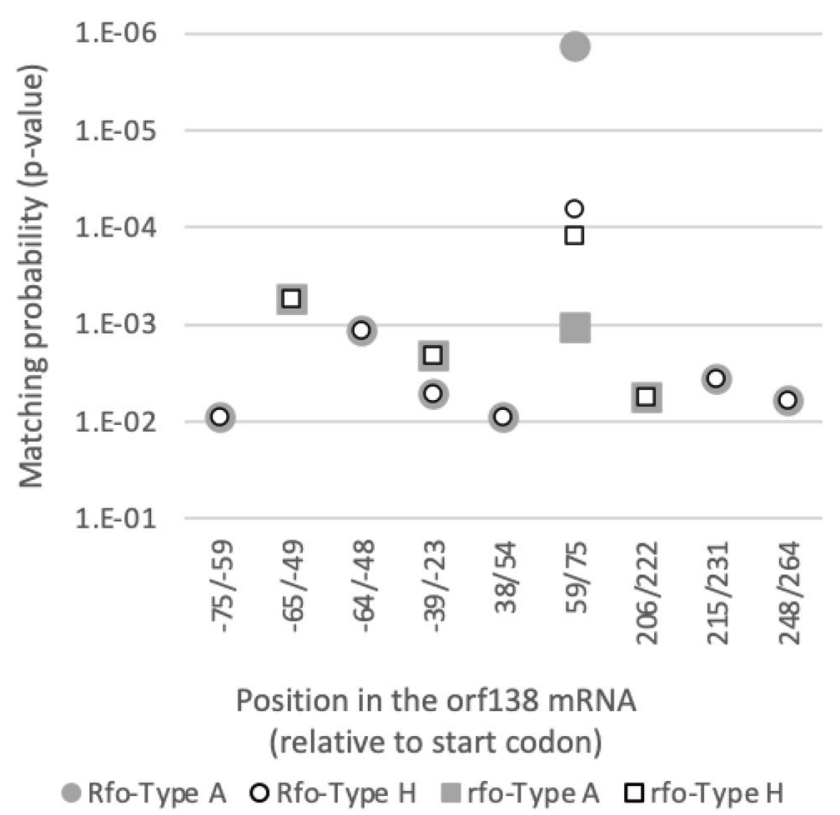

Fig. 4 Putative binding sites of ORF687 in the orf138 mRNA were predicted based on the PPR-code. Matching probability to each putative binding site ( $P$ value) is blotted. ORF687 of 'BK' (Rfo) restores fertility to the Type A orf138 (gray circles), but not to the Type $\mathrm{H}$ (open circle). ORF687 of 'MR' (rfo) cannot restore fertility to either Type A (gray square) or Type H (white square) could not recognize the second nucleotide (U), and the third repeat with a $\mathrm{N}-\mathrm{N}$ combination much preferred the nucleotide $\mathrm{C}$ but not $\mathrm{A}$ in the Type A orf138 (Table 7). Thus, these substitutions induced two and one mismatches with Type A and Type H, respectively (Table 7). Consequently, even if the ORF687 of 'MR' would bind the same 17 nucleotide region, the affinity to orf 138 mRNA was drastically lower than that of 'BK'. The $P$ values were $1.18 \mathrm{E}-04$ with Type $\mathrm{H}$ and $1.04 \mathrm{E}-03$ with Type A (Table 7, Fig. 4). These results clearly explain the reason that 'MR' and 'MS-G' are male sterile. The ORF687 product from $r f o$ allele decreases affinity to the orf $138 \mathrm{mRNA}$ due to the amino acid substitutions and could not suppress the translation of orfl38.

In summary, our results strongly suggested that the ORF687 from 'BK' and 'SK' blocks the translation of Type A orf 138 mRNA by tightly binding to the mRNA in the coding region. However, the single nucleotide substitution from $\mathrm{A}$ in Type $\mathrm{A}$ to $\mathrm{C}$ in Type $\mathrm{H}$ at the 61 st site lowered the binding affinity to the ORF687 and rendered the ORF687 ineffective for the suppression of orf138. On the other hand, the two amino acid substitutions in the ORF687 of 'MR' and 'MS-G' altered the PPR codes and diminished affinity to orf138 mRNA. By these mutations, 'MR' and 'MS-G' 
having the rforfo genotype lost function as the restorer to Ogura CMS.

\section{Discussion}

Here, we found evidence that the function of ORF687 protein, which is encoded by the $R f o$ gene, is to directly bind the mRNA of 17-nucleotides specific sequence in the coding region of orf138. The direct attachment of ORF687 to orf 138 mRNA disrupts the translation of orf138. Two amino acid substitutions at the 5th of the second and third PPR motifs alter sequence specificity of the ORF687 and determine the alleles to be either $R f o$ with the restoring ability or $r f o$ which lacks the function. In the nucleotide

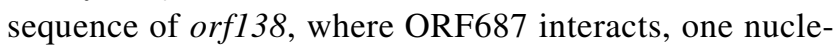
otide substitution is present between Type A and Type $\mathrm{H}$. Because of the substitution, the binding affinity of ORF687 to Type $\mathrm{H}$ transcript is significantly decreased, and ORF687 becomes ineffective. Our results also suggested that some cultivated radishes possess another Rf gene other than $R f o$ and $R f t$. The product of the new Rf gene named $R f s$ processes the orf $138 \mathrm{mRNA}$ at around the 156th-159th nucleotide from the start of the coding sequence. It is of interest that Type $\mathrm{A}$ and Type $\mathrm{H}$ of orf138 show different responses to $R f s$, and further, that these responses are opposite of what was observed for their interactions with ORF687.

\section{Distribution of orf138 haplotypes}

We observed, in our preliminary survey, that more than 10 radish varieties sold in Japan showed male sterility and all of them had orf138. The orf138 of most varieties were Type A, while a few including ' $M R$ ' and ' $\mathrm{BK}$ ' used in this experiment had Type $\mathrm{H}$ orf138. The orf138 whose sequence was published hitherto (Bonhomme et al. 1992; Krishnasamy and Makaroff 1993), and used worldwide for $F_{1}$ breeding is Type A (Yamagishi and Terachi 2001). But Type A is not the major one in Japanese wild radishes and $R$. raphanistrum. Instead, Type B was mainly distributed both in Japanese wild radishes and $R$. raphanistrum (Yamagishi and Terachi 2001). Based on the distribution and the numbers of nucleotide variations among the types, we inferred that Type B is an ancestral form of orf 138 (Yamagishi and Terachi 2001). Type A orf138 is different from Type B by a single synonymous change at the 99th site, and Type $\mathrm{H}$ has a single non-synonymous nucleotide substitution at the 61 st site compared to Type B. The relationships between Type A orf138 or Type H orf138 and $\mathrm{Rf}$ genes were investigated in this article. It would be important to reveal the responses of Type B orfl 38 to the Rf genes. In addition, though Type A was observed in Japanese wild radishes, Type $\mathrm{H}$ was unique to Taiwanese cultivars and was not found in wild radishes in our previous study (Yamagishi and Terachi 2001). Thus, the origin of Type $\mathrm{H}$ orf 138 of Taiwanese and Japanese cultivars remains to be studied further.

\section{Two amino acid substitutions in rfo allele affect the PPR code for ORF687}

This is the first report to clarify the correlations between a nucleotide substitution in a mitochondrial CMS gene and the function of an Rf gene. The majority of Rf genes cloned to date encode proteins belonging to the P-type PPR protein family (Dahan and Mireau 2013). The P-type PPR proteins consist of 2-30 repeats of 35 amino acid length double helix motifs and associate with single strand RNA in a sequence specific manner. So far, annotated P-type PPR proteins participate in various aspects of organellar gene expression including transcription, RNA stabilization, $5^{\prime}$ processing, intron splicing, RNA editing, and translation (Barkan and Small 2014). The Rf gene ( $R f o$ ) of Ogura CMS encodes a member of the P-type PPR protein family (Brown et al. 2003; Desloire et al. 2003; Koizuka et al. 2003). Of the three reports, two of them, Brown et al. (2003) and Koizuka et al. (2003), described that the PPR encoded by the Rfo gene consists of 16 repeats, 15 comprised 35 amino acids and one comprised 36 amino acids. In the third study, Desloire et al. (2003) described that the PPR comprised 15 repeats of 35 amino acids, one of 36 amino acids and one with 46 amino acids. Later, Qin et al. (2014) found an additional PPR motif at the N-terminus using a web-based tool kit, TPRpred (Karpenahalli et al. 2007). In this report, we did not adopt the N-terminal sequence as a PPR motif, since recently published PPR motif prediction tools did not define it (Yan et al. 2019) (Table 7).

The amino acid substitution at the 118th position, which was postulated to have an important role for $\mathrm{Rf}$ function (Imai et al. 2002) is located at the 5th of the second motif. Interestingly, the 153rd amino acid, for which Imai et al. (2002) also found a substitution, was also present at the 5th position of the third motif (Table 7). These observations coincide with the prediction by Koizuka et al. (2003) that the second and third PPR motifs are essential for fertility restoration. PPR proteins function as sequence-specific singlestranded RNA binding proteins, and one RNA base coordinates with one PPR motif (Shen et al. 2015). To recognize a specific nucleotide, a combinatorial amino acid code in each PPR motif plays a key role (PPR-code). The key positions that confer RNA specificity were originally defined as the 6th amino acid of one repeat element and the first amino acid at the C-terminally adjacent repeat ( 6 and 1') (Barkan et al. 2012; Barkan and Small 2014), while the same positions 
were annotated as 4 and ii by Yagi et al. (2013). Furthermore, following the structural analyses of PPR10, the same combinatorial di-residues were called the 5 th and the 35 th (Yan et al. 2019). These various numbering systems for the two crucial amino acid positions rely on the distinct definitions of a PPR unit in a PPR domain. Nevertheless, it is of much interest that both the 118th and the 153rd amino acid are placed at the 5th position (with the latest definition) of PPR motifs and function in nucleotide recognition (Table 7).

\section{Invalidation of ORF687 in 'BK' is due to neither the different sequence nor lower expression level of $R$ fo locus}

The region containing the $R f o$ locus was described to consist of three PPR genes called Ppr-A, Ppr-B and a pseudogene Ppr-C (Desloire et al. 2003). PPR-A and PPR-B are composed of 686 and 687 amino acids, respectively, but the Ppr$B$ was assigned to the $R f o$ gene since only it acts as a fertility restorer gene. Crossing-over in the region around $\mathrm{Rf}$ loci has often made analysis of the Rf gene very difficult. For example, Wang et al. (2010) identified a chimeric gene that resulted from an inter-genic recombination between the two PPR loci. Such recombination created the rf type PPR gene even though the coding sequence for the PPR was identical to the Rf type. The rf type radish possessed the combination of a promoter region from a non-restoring allele and the coding region from the $\mathrm{Rf}$ type, and the PPR gene was not transcribed (Wang et al. 2017). Whereas in the Rf genes of petunia, that was the first cloned PPR protein coding gene, the difference between the fertility restoring genotype and the non-restoring genotype was in the promoter region, not in the coding sequence of the PPR protein (Bentolila et al. 2002). With this in mind, we compared the transcription of $R f o$ in fertile and sterile progeny plants; however, we found that $R f o$ was transcribed at equally high levels in both fertile and sterile plants (Table 6). This result allowed us to conclude that the reason for the failure of fertility restoration in the sterile plants resulting from the cross of ' $\mathrm{MR}$ ' $\times$ (' $\mathrm{BK}$ ' $\times$ ' $S K$ ') was not by the different transcription level of the Rfo gene.

\section{ORF687 directly binds the coding region of orf138 to prevent translation}

It has been already demonstrated that the accumulation of full-length orf138 mRNA is not affected in the fertilityrestored plants by ORF687. Thus, ORF687 must alter the expression of orf138 at the post-transcriptional level. But the precise molecular mechanisms of the suppression have remained unknown. It had been described that the orfl38 transcripts were translated with the same efficiency in sterile and fertility-restored plants, and the ORF687 affected the post-translational stability of the ORF138 protein (Bellaoui et al. 1999). However, Uyttewaal et al. (2008) demonstrated that the primary role of ORF687 in restoring fertility was to inhibit ORF138 synthesis via its ability to directly or indirectly interact with the orf138 RNA and postulated that the role of ORF687 was translational regulation of orf138 mRNA. They proposed that ORF687 could associate with the 5' UTR of orf138 mRNA and prevented either the attachment or the progression of the mitochondrial translation machinery (Uyttewaal et al. 2008).

Our sequence analysis clarified that Type A and Type $\mathrm{H}$ orf138 have identical $5^{\prime}$ processing sites as well as sequences in the 5' UTR of orf138. Therefore, the association of ORF687 with the 5' UTR of orf138 mRNA proposed by Uyttewaal et al. (2008) cannot explain why ORF687 does not function to Type H orf138. Furthermore, our computational prediction strongly suggested that the association of ORF687 with orf138 mRNA is present in the coding region. The 17 PPR motifs bind the 17 nucleotide-length target sequence in the mRNA, and the third position of the target sequence corresponds to the 61st nucleotide of orf138 where Type A and Type $\mathrm{H}$ have $\mathrm{A}$ and $\mathrm{C}$, respectively (Tables 2, 7). The 5th and 35 th amino acid combination affecting the PPR code of the third motif is threonine and asparagine in 'BK' and 'SK', which strongly correlates with A (Shen et al. 2015). Therefore, the ORF687 strongly binds Type A orf138, which has A here, and consequently mitochondrial translational machinery somehow cannot function. In contrast, by the nucleotide substitution at the 61st site from A to C, the affinity of ORF687 to the target sequence is decreased in Type H, and the translation of orf138 mRNA is not disturbed by ORF687. This scenario was further supported by the inactive ORF687 in the rfo allele. The second and third PPR motifs of the Rfo type ORF687 have high affinity to the 17 nucleotide targets in the Type A, while those in rfo type ORF687 have T-D and N-N combinations, respectively, and their nucleotides preference changes from UA to GC (Table 7). Accordingly, the rfo type ORF687 has less affinity to the 17 nucleotides target sequence in Type A as well as that in Type $\mathrm{H}$ orf138 mRNA (Fig. 4). These genetic and PPR-code-based computational analyses strongly suggest that the function of ORF687 is to directly interact with orf138 mRNA in the coding region and to suppress the translation of $\operatorname{orf} 138$.

\section{How does ORF687 suppress translation of ORF138?}

Although our results strongly suggested that ORF687 directly binds to the coding region of orf138, it is unclear how the PPR-RNA interaction leads to suppression of translation. Several PPR proteins affecting translation in plant organelles have been reported (Manna 2015). Only a few such PPR proteins have been analyzed for their target 
sequences. PPR10 and PGR3 bind adjacent to the translation initiation site at atpH and pet $L$, respectively, and resolve local stem-loop structures that would prevent the binding of ribosomes (Prikryl et al. 2011; Fujii et al. 2013). In contrast, ORF687 works as a negative regulator for translation. So far no PPR proteins which negatively regulate the mRNA specific translation have been described except for the ORF687. Secondary structure prediction of the orf138 mRNA did not find a stem-loop including the start codon but found a stem-loop including another AUG, which is located 5 nucleotides upstream of the initiation codon. The stemloop seems to suppress the translation from the upstream AUG and accordingly induces translation from the downstream AUG. If translation started from the upstream AUG, the translation would stop at a UAG overlapped with the initiation codon of the ORF138. Thus, translation from the downstream AUG would be suppressed. Binding of ORF687 to the 17-nucleotide target sequence in the coding region may rearrange secondary structure of the orf 138 mRNA and indirectly resolve the $5^{\prime}$ short stem-loop. It would be also possible that ORF687 work as a decoy for other RNA binding proteins, which bind around the translation initiation site and prevent translation of ORF138. Alternatively, stick binding of ORF687 to the target sequence might just physically impede moving of ribosomes at the site. In any case, further investigation will be necessary for understanding the molecular mechanism of translational suppression of ORF138 by ORF687. A PPR protein-mediated translational suppression is a new concept of gene regulation and will be another powerful tool to control expression of a given gene in plant organelles.

\section{A new Rf gene that processes orf138 mRNA}

In the progeny from the cross of 'MR' $\times($ ' $\mathrm{BK}$ ' $\times$ 'SK'), all of the fertile plants showed complete processing of the orf138 mRNA in the coding region (Table 4). Many PPR proteins belonging to the restorer of fertility-like subfamily are often involved in 5' end formation of mitochondrial transcripts in Arabidopsis (Jonietz et al. 2010; Stoll et al. 2014; Arnal et al. 2014). The Rft product also functioned in $5^{\prime}$ end formation (Yasumoto et al. 2009), but the processing sites were different from those observed in the progeny of 'MR' $\times($ 'BK' $\times$ 'SK'). This finding indicates that 'SK' has a new and third Rf gene, different from $R f o$ and $R f t$. We named this Rf gene $R f s$, and are planning to identify it. The processing sites by the product of $R f s$ varied clone-to-clone but were closely located to those observed for Type B orf 138 of the European wild radish ( $R$. raphanistrum) (Giancola et al. 2007) (Fig. 3). The European Ogura related cytoplasm (Type B orf138) did not cause sterility, and it was interpreted to be due to cytoplasmic suppression of male sterility (Giancola et al. 2007). The similar processing sites at around the 156th-159th from the AUG initiation codon observed in the progeny of 'MR' $\times($ ' $\mathrm{BK}$ ' $\times$ 'SK') strongly suggests that the European wild radish and 'SK' encode the same Rf gene. Unfortunately, Giancola et al. (2007) did not continue the project; however, the corresponding author of that study has suggested that their intriguing observation is attributable to one of the Rf loci present in wild radishes (Budar, personal communication). The new $\mathrm{Rf}$ gene, $R f s$, found here has the ability to process the Type $\mathrm{H}$ orfl 38 mRNA. However, it cannot process the Type A orf 138 mRNA of 'MS-G' (Table 5, Fig. 2). Type A orf138 has A at the 99th nucleotide site where Type B and Type $\mathrm{H}$ have $\mathrm{G}$ (Table 2). It is necessary to clone $R f s$ and determine the amino acid sequence of the product in order to further understand the correlations between $R f s$ and the types of orf138 at a molecular level.

Supplementary Information The online version contains supplementary material available at https://doi.org/10.1007/s00438-021-01777-y.

Acknowledgements We are grateful to Brody Frink for providing language help. This work was supported by the fund of Research Center of Botany, Kyoto Sangyo University to H. Y. and by JSPS Gants-in-Aid for Scientific Research (18H02462 to M. T.).

Author contributions HY and TT: designed research; HY, MJ, KO, $\mathrm{AH}$ and AF: performed research; HY, AH and MT: analyzed data; HY and MT: wrote the paper.

Funding This work was supported by the fund of Research Center of Botany (F2001), Kyoto Sangyo University to H.Y. and by JSPS Grantsin-Aid for Scientific Research (18H02462 to M.T.).

\section{Declarations}

Conflict of interest Authors declare no conflicts of interest.

Ethical approval This article does not contain any studies with human participants or animals.

Consent to participate All the authors consent to participate in this work.

Consent for publication All the authors agree the publication.

Open Access This article is licensed under a Creative Commons Attribution 4.0 International License, which permits use, sharing, adaptation, distribution and reproduction in any medium or format, as long as you give appropriate credit to the original author(s) and the source, provide a link to the Creative Commons licence, and indicate if changes were made. The images or other third party material in this article are included in the article's Creative Commons licence, unless indicated otherwise in a credit line to the material. If material is not included in the article's Creative Commons licence and your intended use is not permitted by statutory regulation or exceeds the permitted use, you will need to obtain permission directly from the copyright holder. To view a copy of this licence, visit http://creativecommons.org/licenses/by/4.0/. 


\section{References}

Arnal N, Quadrado M, Simon M, Mireau H (2014) A restorer-offertility like pentatricopeptide repeat gene directs ribonucleolytic processing within the coding sequences of rps3-rpl16 and orf $240 a$ mitochondrial transcripts in Arabidopsis thaliana. Plant J 78:134-145

Barkan A, Small I (2014) Pentatricopeptide repeat protein in plants. Annu Rev Plant Biol 65:415-442

Barkan A, Rojas M, Fujii S, Yap A, Chang YS et al (2012) A combinatorial amino acid code for RNA recognition by pentatricopeptide repeat proteins. PLoS Genet 8:e1002910

Bellaoui M, Grelon M, Pelletier G, Budar F (1999) The restorer Rfo gene acts post-translationally on the stability of the ORF138 Ogura-associated protein in reproductive tissue of rapeseed cybrids. Plant Mol Biol 40:893-902

Bentolila S, Alfonso AA, Hanson MR (2002) A pentatricopeptide repeat-containing gene restores fertility to cytoplasmic malesterile plants. Proc Natl Acad Sci USA 99:10887-10892

Bonhomme S, Budar F, Féraut M, Pelletier G (1991) A 2.5 kb NcoI fragment of Ogura radish mitochondrial DNA is correlated with cytoplasmic male-sterility in Brassica cybrids. Curr Genet 19:121-127

Bonhomme S, Budar F, Lancelin D, Small I, Defrance M-C, Pelletier G (1992) Sequence and transcript analysis of the Nco2.5 Oguraspecific fragment correlated with cytoplasmic male sterility in Brassica cybrids. Mol Gen Genet 235:340-348

Brown GG, Formanová N, Jim H, Wargachuk R, Dendy C, Patil P, Laforest M, Zhang J, Cheng WY, Landy BS (2003) The radish Rfo restorer gene of Ogura cytoplasmic male sterility encodes a protein multiple pentatricopeptide repeats. Plant J 35:262-272

Chen L, Liu Y-G (2014) Male sterility and fertility restoration in crops. Annu Rev Plant Biol 65:579-606

Dahan J, Mireau H (2013) The Rf and Rf-like PPR in higher plants, a fast-evolving subclass of PPR genes. RNA Biol 10:1469-1476

Desloire S, Gherbi H, Laloui W, Marhadour S, Clouet V et al (2003) Identification of the fertility restoration locus, $R f o$, in radish as a member of the pentatricopeptide-repeat protein family. EMBO Rep 4:588-594

Fujii S, Sato N, Shikanai T (2013) Mutagenesis of individual pentaticopeptide repeat motifs affects RNA binding activity and reveals functional partitioning of Arabidopsis PROTON gradient reguration3. Plant Cell 25:3079-3088

Gaborieau L, Brown GG, Mireau H (2016) The propensity of pentatricopeptide repeat genes to evolve into restorer of cytoplasmic male sterility. Front Plant Sci 7:1816

Giancola S, Rao Y, Chaillou S, Hiard S, Martin-Canadell A, Pelletier G, Budar F (2007) Cytoplasmic suppression of Ogura cytoplasmic male sterility in European natural populations of Raphanus raphanistrum. Theor Appl Genet 114:1333-1343

Hanson MR, Bentolila S (2004) Interactions of mitochondrial and nuclear genes that affect male gametophyte development. Plant Cell 16(Suppl 1):S154-S169

Imai R, Koizuka N, Fujimoto H, Hayakawa T, Kimura Y, KohnoMurase J, Sakai T, Imamura J (2002) Identification and characterization of a fertility restorer gene, $\mathrm{rfk} 1$, for Kosena cms. (3) Analysis of allelic polymorphism relevant to rfk1 gene function. Breed Res 4(Suppl. 2):184 (In Japanese)

Jonietz C, Forner J, Hölzle A, Thuss S, Binder S (2010) RNA PROCESSING FACTOR 2 is required for 5 ' end processing of $\operatorname{nad} 9$ and cox3 mRNAs in mitochondria of Arabidopsis thaliana. Plant Cell 22:443-453

Karpenahalli MR, Lupas AN, Soding J (2007) TPRpred: a tool for prediction of TPR-, PPR- and SEL1-like repeats from protein sequences. BMC Bioinform 8:2
Koizuka N, Imai R, Fujimoto H, Hayakawa T, Kimura Y, KohnoMurase J, Sakai T, Imamura J (2003) Genetic characterization of a pentatricopeptide repeat protein gene, orf687, that restores fertility in the cytoplasmic male-sterile Kosena radish. Plant J 34:407-415

Krishnasamy S, Makaroff CA (1993) Characterization of the radish mitochondrial orfB locus: possible relationship with sterility in Ogura radish. Curr Genet 24:156-163

Laser KD, Lersten NR (1972) Anatomy and cytology of microsporogenesis in cytoplasmic male sterile angiosperms. Bot Rev $38: 425-454$

Manna S (2015) An overview of pentatricopeptide repeat protein and their applications. Biochimie 113:93-99

Ogura H (1968) Studies on the new male sterility in Japanese radish, with special reference to the utilization of this sterility towards the practical raising of hybrid seeds. Mem Fac Agric Kagoshima Univ 6:39-78

Perrin R, Meyer EH, Zaepfel M, Kim Y-J, Mache R, Grienenberger J-M, Gualberto M, Gagliardi D (2004) Two exoribonucleases act sequentially to process mature 3'-ends of atp 9 mRNAs in Arabidopsis mitochondria. J Biol Chem 229:25440-25446

Prikryl J, Rojas M, Schuster G, Barkan A (2011) Mechanism of RNA stabilization and translational activation by a pentatricopeptide repeat protein. Proc Natl Acad Sci USA 108:415-420

Qin X, Warguchuk R, Arnal N, Gaborieau L, Mireau H, Brown GG (2014) In vivo functional analysis of a nuclear restorer PPR protein. BMC Plant Biol 14:313

Shen C, Wang X, Liu Y, Li Q, Yang Z, Yan N, Zou T, Yin P (2015) Specific RNA recognition by designer pentatricopeptide repeat protein. Mol Plant 8:667-670

Stoll B, Zendler D, Binder S (2014) RNA processing factor 7 and polynucleotide phosphorylase are necessary for processing and stability of $n a d 2$ mRNA in Arabidopsis mitochondria. RNA Biol 11:968-976

Uyttewaal M, Arnal N, Quadrada M, Martin-Canadell A, Vrielynac N, Niard S, Gherbi H, Bendahmane A, Budar F, Mireau H (2008) Characterization of Raphanus sativus pentatricopeptide repeat proteins encoded by the fertility restorer locus for Ogura cytoplasmic male sterility. Plant Cell 20:3331-3345

Wang ZW, Zhang LT, Chen J, Xiang PC, Mei SK, Zhou Y, Wang T (2010) A chimeric Rfo gene generated by intergenic recombination cosegregates with the fertility restorer phenotype for cytoplasmic male sterility in radish. Mol Breed 25:339-349

Wang ZW, Wang CD, Cai QZ, Mei SY, Gao L, Zhou Y, Wang T (2017) Identification of promoter exchange at a male fertility restorer locus for cytoplasmic male sterility in radish (Raphanus sativus L.). Mol Breed 37:82

Yagi Y, Hayashi S, Kobayashi K, Hirayama T, Nakamura T (2013) Elucidation of the RNA recognition code for pentatricopeptide repeat proteins involved in organelle RNA editing in plants. PLoS ONE 8:e57268

Yamagishi H, Terachi T (1996) Molecular and biological studies on male-sterile cytoplasm in the Cruciferae. III. Distribution of Ogura-type cytoplasm among Japanese wild radishes and Asian radish cultivars. Theor Appl Genet 93:325-332

Yamagishi H, Terachi T (1997) Molecular and biological studies on male-sterile cytoplasm in the Curuciferae. IV. Ogura-type cytoplasm found in the wild radish, Raphanusa raphanistrum. Plant Breed 116:323-329

Yamagishi H, Terachi T (2001) Intra- and inter-specific variations in the mitochondrial gene orf 138 of Ogura-type male-sterile cytoplasm from Raphanus sativus and Raphanus raphanistrum. Theor Appl Genet 103:725-732

Yan J, Yao Y, Hong S, Yang Y, Shen C, Zhang Q, Zhang D, Zou T, Yim $P$ (2019) Delineation of pentatricopeptide repeat codes for target RNA prediction. Nucleic Acids Res 47:3728-3738 
Yasumoto K, Matsumoto Y, Terachi T, Yamagishi H (2008) Restricted distribution of orf687 as the pollen fertility restorer gene for Ogura male sterility in Japanese wild radish. Breed Sci 58:177-182

Yasumoto K, Terachi T, Yamagishi H (2009) A novel $R f$ gene controlling fertility restoration of Ogura male sterility by RNA processing of orf138 found in Japanese wild radish and its STS markers. Genome 52:495-504
Publisher's Note Springer Nature remains neutral with regard to jurisdictional claims in published maps and institutional affiliations. 